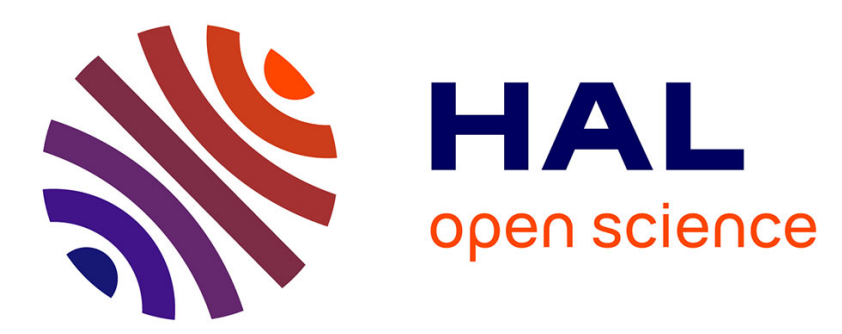

\title{
Using multimodal MR data for segmentation and topology recovery of the cerebral superficial venous tree
}

Nicolas Passat, Christian Ronse, Joseph Baruthio, Jean-Paul Armspach, Marcel Bosc, Jack Foucher

\section{- To cite this version:}

Nicolas Passat, Christian Ronse, Joseph Baruthio, Jean-Paul Armspach, Marcel Bosc, et al.. Using multimodal MR data for segmentation and topology recovery of the cerebral superficial venous tree. International Symposium on Visual Computing (ISVC), 2005, Lake Tahoe, United States. pp.60-67, 10.1007/11595755_8. hal-01694970

\section{HAL Id: hal-01694970 \\ https://hal.univ-reims.fr/hal-01694970}

Submitted on 31 Jan 2018

HAL is a multi-disciplinary open access archive for the deposit and dissemination of scientific research documents, whether they are published or not. The documents may come from teaching and research institutions in France or abroad, or from public or private research centers.
L'archive ouverte pluridisciplinaire HAL, est destinée au dépôt et à la diffusion de documents scientifiques de niveau recherche, publiés ou non, émanant des établissements d'enseignement et de recherche français ou étrangers, des laboratoires publics ou privés. 


\title{
Using multimodal MR data for segmentation and topology recovery of the cerebral superficial venous tree
}

\author{
N. Passat ${ }^{1,2}$, C. Ronse ${ }^{1}$, J. Baruthio ${ }^{2}$, J.-P. Armspach ${ }^{2}$, M. Bosc ${ }^{3}$, and \\ J. Foucher ${ }^{4}$ \\ 1 LSIIT, UMR 7005 CNRS-ULP, Strasbourg 1 University, France \\ 2 IPB-LNV, UMR 7004 CNRS-ULP, Strasbourg 1 University, France \\ 3 L2TI, EA 3043, Paris 13 University, France \\ 4 INSERM U405, France
}

\begin{abstract}
Magnetic resonance angiography (MRA) produces 3D data visualizing vascular structures by detecting the flowing blood signal. While segmentation methods generally detect vessels by only processing MRA, the proposed method uses both MRA and non-angiographic (MRI) images. It is based on the assumption that MRI provides anatomical information useful for vessel detection. This supplementary information can be used to correct the topology of the segmented vessels. Vessels are first segmented from MRA while the cortex is segmented from MRI. An algorithm, based on distance maps and topology preserving thinning, then uses both segmented structures for recovery of the missing parts of the brain superficial venous tree and removal of other vessels. This method has been performed and validated on 9 MRA/MRI data of the brain. The results show that the venous tree is correctly segmented and topologically recovered with a $84 \%$ accuracy.
\end{abstract}

\section{Introduction}

Magnetic resonance angiography (MRA) proposes non-invasive and non-irradiant techniques providing 3D images of flowing blood. These techniques are frequently used to study the vascular structures of the brain. Indeed, the availability of precise information about brain vascular networks is fundamental for detecting pathologies or to use the vessels as anatomical landmarks.

Despite the development of numerous methods to perform blood vessel segmentation of 3D angiographic data (see [1] for an overview), there is still none that is able to provide completely satisfactory results. In order to improve the segmentation accuracy, methods have been designed to process multimodal angiographic data (DSA and MRA in [2], or DSA and IVUS in [3]), taking advantage of the supplementary information provided by each image. More recently, a new kind of vessel segmentation strategy involving angiographic and nonangiographic data has been developed. This new approach enables to consider a priori knowledge concerning relations between vessels and non-vascular structures, and to use this knowledge for guidance of segmentation algorithms. The 
first method based on this approach has been proposed in [4] for segmentation of the superior sagittal sinus from cerebral MRA and T1 MRI.

In this paper, a new segmentation method devoted to the cerebral superficial venous tree is proposed. The main purpose of this method is to provide a correct representation of this structure prior to transcranial stimulation procedures. For such procedures, an accurate segmentation of the cerebral superficial venous tree is fundamental, since vessels can be used as landmarks for determining specific cerebral areas. More especially, it is important that the segmented tree presents correct topological properties.

The method described in this paper is based on a preliminary step, providing segmentation of brain vessels. It then uses a priori knowledge about relations between cerebral vessels and the cortex to discriminate superficial venous structures from other vessels and to recover a topologically correct representation of the whole cerebral superficial venous tree. This method is inspired from a strategy based on topological thinning proposed in [5], but is however quite new since it uses multimodal angiographic and non-angiographic data, and high level anatomical knowledge for guidance of the segmentation process.

This paper is organized as follows. In Section 2, related work on topology preserving vessel segmentation is discussed. The main purpose leading to the method proposed in this paper is then detailed, justifying the chosen strategy. The successive steps of the method are described in Section 3. In Section 4, the method is tested and analyzed on a 9 image database. Discussion and further works are presented in Section 5.

\section{Related Work and Purpose}

Angiographic data acquisition processes such as MRA or computed tomography angiography (CTA) enable to visualize complex 3D vascular structures. Several methods devoted to vessel segmentation from such 3D data have been proposed. Although few of them can deal with topology modifications (some can detect junctions or bifurcations [6], or can generate a graph of the segmented vessels [7]), nearly none of them have been designed to preserve correct structural properties when applied on complex vascular structures.

To the best of our knowledge, there exists only one method, proposed in [5], enabling to segment a whole vascular tree presenting correct topological properties. By assuming that a vascular tree (i.e. a structure composed of one connected component with no hole and no cavity) is topologically equivalent to the volume of the whole image, it proposes to apply a thinning algorithm starting from this whole image and iteratively removing simple points. This method has been applied for liver vascular tree segmentation from CTA.

A topologically correct segmentation of the cerebral superficial venous tree ${ }^{5}$ can be quite useful for determining specific brain areas for transcranial magnetic

\footnotetext{
5 The whole superficial venous structures of the brain can present cycles generated by anastomoses. However, MRA of the top of the head only visualize a part of them really presenting a tree topology.
} 
stimulation. The angiographic data provided for such applications are generally MRA visualizing vascular structures of the top of the head. Unfortunately, the method proposed in [5] presents many limitations making it inapplicable to MRA. First, it assumes that all the vessels located in the image belong to a same vascular tree. However, this is not true for cerebral images where intra and extracranial veins and arteries are organized in a more complex way. Second, the proposed thinning process is guided by the angiographic data intensity, assuming that the whole vascular tree presents a higher signal than the remaining tissues. This is no longer true for MRA where data signal loss can be caused by turbulences of the blood flow.

A correct segmentation of the cerebral superficial venous tree from MRA then requires to be able to discriminate the vessels belonging to this tree from the other ones, and to recover their correct topology despite signal loss in the angiographic data. A possible way to solve this problem can then consist in using a priori anatomical knowledge concerning these vascular structures. It is proposed here to use knowledge about the spatial relation existing between these searched venous structures and the cortex. This approach requires to determine information not only on vascular structures but also on non-vascular ones. To do so, the method described hereafter uses both MRA and MRI data.

\section{Method}

\subsection{Input and Output}

The method takes as input two MR data. The first one is a classical MRI of the whole head, denoted by $f_{m r i}: I \rightarrow \mathbb{Z}$ (with $I \subset \mathbb{Z}^{3}$ ). The second one is a MRA of the top of the head, only containing information about the flowing blood, denoted by $f_{m r a}: I \rightarrow \mathbb{Z}$. Both MRA and MRI have to be correctly registered (if they were not generated during the same image acquisition, a rigid registration is sufficient since both images correspond to the same patient). In the proposed application, the used images are T1 MRI and time-of-flight (TOF) MRA of the top of the head which are already correctly superimposed (Fig. 1 ). The method provides as output a binary image $V_{\text {seg }} \subset I$ of the segmented cerebral superficial venous tree.

\subsection{Preliminary Step: Vessel and Brain Segmentation}

This first step consists in computing preliminary segmentations of the vessels from $f_{m r a}$ and of the brain from $f_{m r i}$ (middle and left parts of Fig. 2).

All the vessels (i.e. the flowing blood) visualized in $f_{m r a}$ are segmented using a region-growing method described in [8]. The obtained segmentation is a set $V_{i n i} \subset I$, assumed not to contain false positives, composed of several connected components corresponding to deep cerebral vessels, superficial venous vessels and scalp vessels. It has to be noticed that these components do not contain any holes or cavities, since the region-growing segmentation uses topologically simple points. 

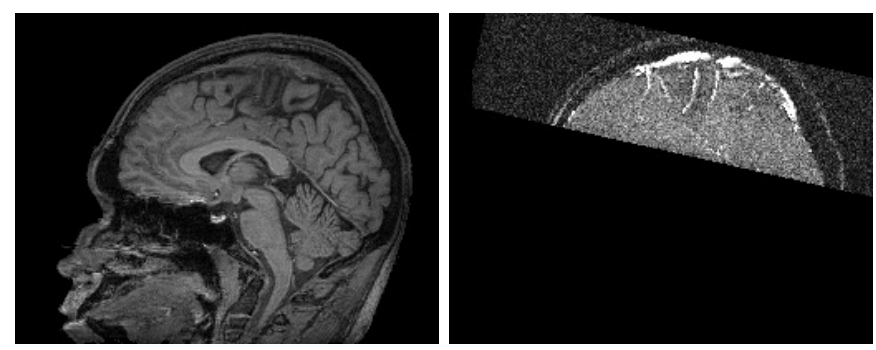

Fig. 1. Sagittal slices of the processed data. Left: T1 MRI of the whole head. Right: TOF MRA of the top of the head.

The brain is segmented from $f_{m r i}$, using an energy minimization method proposed in [9]. This method discriminates the intracranial volume into three categories corresponding to white matter (WM), gray matter (GM) and cerebrospinal fluid (CSF). The result $\left(C_{C S F}, C_{G M}, C_{W M}\right) \subset I^{3}$ is composed of three sets respectively corresponding to these three kinds of tissues.

\subsection{Vascular Tree Reconstruction}

Vessel Labeling Let $V_{\text {seg }} \subset I$ be the vascular tree being searched ( $V_{\text {seg }}$ has to present a tree topology, being composed of one connected component with no hole and no cavity). Correct initialization and termination of the vascular tree reconstruction require to determine two sets $V_{\max }, V_{\min } \subset I$ such as:

$$
V_{\text {min }} \subseteq V_{\text {seg }} \subseteq V_{\max }
$$

with $V_{\max }$ having the same topology as $V_{\text {seg }}$ and $V_{\min }$ containing no hole and no cavity. As already proposed in [5], it is possible to assume that $V_{\max }=I$. The determination of $V_{\min }$ is now described.

The set $V_{\min }$ is assumed to only contain vascular structures which will constitute the basis for the topology recovery procedure. The preliminary segmentation step provides a set $V_{i n i}$ containing all the vessels of $f_{m r a}$. However, $V_{i n i}$ is composed of connected components corresponding not only to superficial venous vessels but also to scalp and deep cerebral vessels. A vessel labeling procedure is then necessary to determine the vessels belonging to $V_{\text {ini }}$.

Several assumptions can be made about the position of these vessels with respect to the different brain structures. Indeed, deep cerebral structures are located far from the skull (i.e. far from the external border of the CSF). Moreover, superficial venous vessels are located close to the cortex (i.e. close to the external border of the GM), while scalp vessels are located far from it.

In order to classify the connected components of $V_{i n i}$, two distance maps are computed. The first one $\left(d_{C S F}\right)$ indicates the signed distance to the external border of the set $C_{C S F}$ (distances positive inside the brain and negative outside). The second one $\left(d_{G M}\right)$ indicates the signed distance to the external border of 

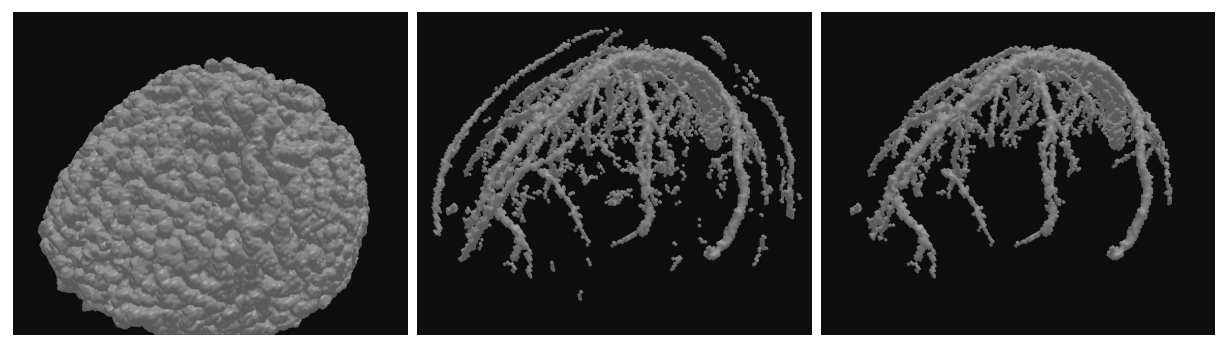

Fig. 2. Left: gray matter $\left(C_{G M}\right)$ segmented from $f_{m r i}$. Middle: vessels $\left(V_{i n i}\right)$ segmented from $f_{m r a}$. Right: segmented vessels belonging to the cerebral superficial venous tree $\left(V_{\text {min }}\right)$.

the set $C_{G M}$ (distances positive outside the brain and negative inside). For each connected component $v_{i n i} \subset V_{i n i}$, the distance $d$ to the CSF (resp. to the GM) is defined as the minimal value between the CSF (resp. the GM) and any voxel of $v_{\text {ini }}$ :

$$
d\left(C_{\alpha}, v_{i n i}\right)=\min \left\{d_{\alpha}(p) \mid p \in v_{\text {ini }}\right\},(\text { with } \alpha=\mathrm{CSF} \text { or GM }) .
$$

The set $V_{\min }$ (right part of Fig. 2) can then be defined by:

$$
V_{\text {min }}=\bigcup_{d\left(C_{C S F}, v_{i n i}\right)<20} \bigcup_{\mathrm{mm} \text { and } d\left(C_{G M}, v_{i n i}\right)<5 \mathrm{~mm}} v_{i n i} .
$$

The threshold values of this formula have been established for brains presenting a volume of $1200 \mathrm{~cm}^{3}$. They can then be adapted to the size of any brain by applying a correction coefficient $\sqrt[3]{V / 1200}$ where $V \mathrm{~cm}^{3}$ is the current brain volume estimated from $C_{G M}$ and $C_{W M}$.

Topology Recovery The topology recovery step consists in determining the vascular tree $V_{\text {seg }}$ which has to contain the segmented structures $V_{\min }$ and to present correct topological properties. Although $V_{\min }$ can contain several connected components, $V_{\text {seg }}$ must be composed of only one connected component with no hole and no cavity. This can be done by iteratively removing simple points from $V_{\max }$ until obtaining set uniquely composed of voxels belonging to $V_{\min }$ or being non-simple. Such a strategy then enables to reconnect the different branches of $V_{\min }$. However, it does not guarantee that these branches will be reconnected according to the anatomical reality. In [5], the CTA image was used as a weight function to indicate which voxels had to be removed first. This strategy was justified by the assumption that the likeliness of a voxel to belong to a vessel was proportional to its gray-level intensity. This assumption is no longer true for MRA data where signal losses are frequent, making such a strategy unusable. It is then necessary to define a new weight map modeling a vessel-likeliness measure for each voxel, then providing a correct priority for their removal from $V_{\max }$. 
Still assuming that the vessels of the cerebral superficial venous tree are located at the surface of the cortex, it is proposed to use a weight function $f_{\text {weight }}$ defined as the geodesic distance map providing the distance of each point to the closest connected component of $V_{\min }$ inside the mask $I \backslash\left(C_{G M} \cup C_{W M}\right)$. This weight function $f_{\text {weight }}: I \rightarrow \overline{\mathbb{Z}}$ is then defined by:

$$
f_{\text {weight }}(p)= \begin{cases}0 & \text { if } p \in V_{\text {min }} \\ +\infty & \text { if } p \in C_{G M} \cup C_{W M} \\ d_{g} & \text { otherwise }\end{cases}
$$

where $d_{g}$ is distance of the shortest path between $p$ and $V_{m i n}$, not intersecting $C_{G M} \cup C_{W M}$.

The vascular tree reconstruction can then be summarized as follows.

1. $L=\left\{F_{i}\right\}_{i=\min _{I} f_{\text {weight }}}^{\max _{I} f_{\text {eigh }}}$ ( $L$ is a set of empty FIFO lists).

2. $V_{\text {cur }}=V_{\max }$.

3. For all voxel $p$ belonging to the border of $V_{\text {cur }}, p$ is put in $F_{f_{\text {weight }}(p)}$.

4. While $\exists i \in\left[\min _{I} f_{\text {weight }}, \max _{I} f_{\text {weight }}\right], F_{i} \neq \emptyset$ :

(a) get $p$ from $F_{\max \left\{i \mid F_{i} \neq \emptyset\right\} \text {; }}$

(b) if $p$ is a simple point of $V_{\text {cur }}$ and $p \notin V_{\text {min }}$ then $V_{\text {cur }}=V_{\text {cur }} \backslash\{p\}$ and for all neighbors $q$ of $p$ not already in $F_{f_{\text {weight }}(q)}$, add $q$ to $F_{f_{\text {weight }}(q)}$.

5. $V_{\text {seg }}=V_{\text {cur }}$.

\section{Experiments and Results}

A database of $9 \mathrm{MRI} / \mathrm{MRA}$ cases has been used to validate the efficiency of the proposed algorithm. Both TOF MRA and T1 MRI exams (see Fig. 1) were performed on a 2 Tesla whole-body imager (Tomikon S200 Bruker) using a head transmitter and receiver $\mathrm{RF}$ coil. The algorithm presented in this paper has been implemented by using the ImLib3D [10] open source C++ library. The obtained results, summarized in Tables 1 and 2, have been validated by an expert. An example of segmented vascular tree is illustrated in Fig. 3.

One can observe that the results are quite satisfactory for the labeling step. The set $V_{\min }$ generated by the method then constitutes a reliable basis for the topology recovery. The results obtained for this topology recovery step are also satisfactory, even if not perfect. Most of the vessels have been correctly reconnected. However, approximately $16 \%$ of the reconnections are erroneous. These errors can be classified into two categories. The first ones are composed of false reconnections (i.e. reconnections of vessels which should not be connected). These errors occur when the distance between vessels which should be connected is high (generally more than $30 \mathrm{~mm}$ ). The second ones are composed of reconnections which are topologically correct (i.e. reconnections of vessels which should be connected) but which does not exactly correspond to the real vessel trajectory. This analysis seems to prove that the use of geodesic distance between the vessels allows to correctly recover most of the venous tree topology but is not sufficient to deal with the most difficult cases, corresponding to very tortuous 
vessels and largest signal loss in MRA. A solution could then consist in also considering the signal of vessels, no longer in MRA but in MRI and to fuse it with geodesic distance information to provide a most representative weight map.

Table 1. Validations of the labeling step. Mean values of the classification obtained by the method (Results) compared to the real classification of the connected components (c.c.) of $V_{i n i}$ (Reference).

\begin{tabular}{|l|r|r|r|r|r|r|r|r|}
\hline \multirow{2}{*}{ Set } & \multicolumn{2}{|c|}{ Results } & \multicolumn{2}{|c|}{ Reference } & \multicolumn{2}{|c|}{ False positives } & \multicolumn{2}{|c|}{ False negatives } \\
\cline { 2 - 8 } & voxels & c.c. & voxels & c.c. & voxels & c.c. & voxels & c.c. \\
\hline$V_{\text {min }}$ & 11197 & 15.3 & 11223 & 15.8 & $0(0 \%)$ & $0(0 \%)$ & $26(0.2 \%)$ & $0.5(3.1 \%)$ \\
$V_{\text {ini }} \backslash V_{\text {min }}$ & 2191 & 21.3 & 2165 & 20.8 & $26(1.2 \%)$ & $0.5(2.4 \%)$ & $0(0 \%)$ & $0(0 \%)$ \\
\hline
\end{tabular}

Table 2. Validations of the topology recovery step. From left to right: mean number of reconnections, number and ratio of correct and incorrect reconnections.

\begin{tabular}{|c|c|c|}
\hline Reconnections & Correct & Incorrect \\
\hline 14.3 & $12.1(84.6 \%)$ & $2.2(15.4 \%)$ \\
\hline
\end{tabular}

\section{Conclusion}

This paper has presented a novel method using both MRI and MRA for segmentation and topology recovery of the cerebral superficial venous tree. The algorithm uses a priori anatomical knowledge concerning the spatial relations between vessels and brain structures, to discriminate the cerebral venous tree from non-cerebral vessels and to recover the topology of vessel missing parts. The method has been tested on 9 patients, providing satisfactory results in most cases. The main originality of this work consisted in using both angiographic and non-angiographic images to model anatomical knowledge and integrate information on non-vascular structures in a vessel segmentation process. Further works will consist in improving the reliability of this method by using the MRI signal of vessels as proposed in the previous section. Other methods, taking advantage of multimodality and anatomical a priori knowledge are also being developed for segmentation of the whole cerebral vascular tree.

\section{References}

1. Kirbas, C., Quek, F.: A review of vessel extraction techniques and algorithms. ACM Computing Surveys 36 (2004) 81-121 


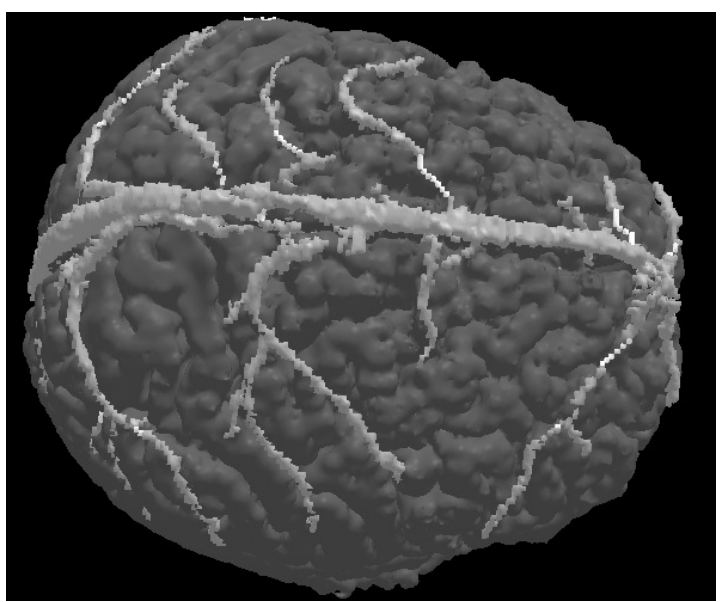

Fig. 3. Cerebral superficial venous tree $\left(V_{\text {seg }}\right)$ segmented by the proposed method. (brain $\left(C_{G M} \cup C_{W M}\right)$ in dark gray, initial segmentation $\left(V_{\text {min }}\right)$ in light gray, topological reconstruction $\left(V_{\text {seg }} \backslash V_{\min }\right)$ in white).

2. Sanderson, A., Parker, D., Henderson, T.: Simultaneous segmentation of MR and $\mathrm{X}$-ray angiograms for visualization of cerebral vascular anatomy. In: International Conference on Volume Image Processing - VIP'93. (1993) 11-14

3. Bloch, I., Pellot, C., Sureda, F., Herment, A.: 3D reconstruction of blood vessels by multi-modality data fusion using fuzzy and Markovian modelling. In: Computer Vision, Virtual Reality and Robotics in Medicine - CVRMed'95. (1995) 392-398

4. Passat, N., Ronse, C., Baruthio, J., Armspach, J.P., Foucher, J.: Using watershed and multimodal data for vessel segmentation: Application to the superior sagittal sinus. In: International Symposium on Mathematical Morphology - ISMM'05. (2005) 419-428

5. Dokládal, P., Lohou, C., Perroton, L., Bertrand, G.: Liver blood vessels extraction by a 3-D topological approach. In: Medical Image Computing and ComputerAssisted Intervention - MICCAI'99. (1999) 98-105

6. Flasque, N., Desvignes, M., Constans, J., Revenu, M.: Acquisition, segmentation and tracking of the cerebral vascular tree on 3D magnetic resonance angiography images. Medical Image Analysis 5 (2001) 173-183

7. Zahlten, C., Jürgens, H., Peitgen, H.O.: Reconstruction of branching blood vessels from CT-data. In: Visualization in Scientific Computing'95, Eurographics Workshop. (1995) 41-52

8. Passat, N., Ronse, C., Baruthio, J., Armspach, J.P., Maillot, C., Jahn, C.: Regiongrowing segmentation of brain vessels: An atlas-based automatic approach. Journal of Magnetic Resonance Imaging 21 (2005) 715-725

9. Bosc, M., Heitz, F., Armspach, J.P.: Statistical atlas-based sub-voxel segmentation of 3D brain MRI. In: International Conference on Image Processing - ICIP'03. (2003) 1077-1080

10. Bosc, M., Vik, T., Armspach, J.P., Heitz, F.: ImLib3D: An efficient, open source, medical image processing framework in $\mathrm{C}++$. In: Medical Image Computing and Computer-Assisted Intervention - MICCAI'03. (2003) 981-982 